\title{
Statistical Validation of the Mixing Length Concept in Bubble Columns Operated in the Transition Flow Regime
}

\author{
Stoyan Nedeltchev and Markus Schubert \\ Institute of Fluid Dynamics, Helmholtz-Zentrum Dresden-Rossendorf, Bautzner Landstraße 400, \\ Dresden 01328, Germany
}

Keywords: Bubble Columns, Flow Regime Identification, Transition Flow Regime, New Statistical Parameter, Mixing Length

\begin{abstract}
A new statistical parameter was defined in order to determine the range of applicability of the mixing length concept in two bubble columns. Gas holdup time series $(60,000$ points) were measured in two bubble columns $(0.15$ and $0.4 \mathrm{~m}$ in i.d.) by a conductivity wire-mesh sensors. The new statistical parameter $\Phi$ was defined as the ratio of the mean value of the signal to three times the average absolute deviation (3AAD) and it was correlated to the mixing length. It was found that this new correlation was not valid in all hydrodynamic regimes. It is applicable only in the transition flow regime. Such a clarification has not been provided in the literature so far. In the narrow bubble column, the results were also confirmed on the basis of the Kolmogorov entropy and another statistical parameter. The new parameter $\Phi$ was also used for flow regime identification. In the narrow bubble column, the first transition velocity $U_{\text {trans }}$ was identified at $0.034 \mathrm{~m} / \mathrm{s}$; whereas, the second $U_{\text {trans }}$ occurred at $0.112 \mathrm{~m} / \mathrm{s}$. In the large bubble column, the two $U_{\text {trans }}$ values were identified at 0.045 and $0.101 \mathrm{~m} / \mathrm{s}$, respectively.
\end{abstract}

\section{Introduction}

Bubble columns are frequently used in chemical, petrochemical and biochemical industries. In spite of the fact that these reactors are used so often and they have been studied so extensively, their accurate design and scale-up is still a difficult multiparameter problem. Bubble columns are vertical gas-liquid contactors, which operate in three different flow regimes: bubbly, transition and churn-turbulent flow. In the case of a coarse gas distributor (such as a perforated plate with large openings), a gas maldistribution regime is observed instead of the bubbly flow regime.

The degrees of mixing, heat and mass transfer as well as the achievable conversion depend on the prevailing flow regime. The main hydrodynamic regimes are characterized with different flow patterns, which depend on gas flow rate, properties of the gas-liquid system, gas distributor design, column diameter, etc. Therefore, it is essential to know the range of parameters over which a particular regime prevails and the conditions under which the transition occurs. For water and dilute aqueous solutions, Shah et al. (1982) have presented a flow regime map (an approximate dependency of flow regime on superficial gas velocity $U_{\mathrm{G}}$ and column diameter). In the past four decades, different measurement techniques have been used for flow regime identification, but the most reliable transition velocities are obtained by means of the noninvasive radioactive techniques combined with nonlinear chaos analysis and information entropy the-

Received on June 11, 2014; accepted on July 18, 2014

DOI: 10.1252 /jcej.14we189

Correspondence concerning this article should be addressed to $\mathrm{S}$. Nedeltchev (E-mail address: s.nedeltchev@hzdr.de). ory (Nedeltchev and Shaikh, 2013).

The bubbly flow (homogeneous) regime is characterized with relatively small bubbles and only gentle agitation. The bubble size distribution is rather narrow and defined by the gas distributor used. Further, a relatively uniform cross-sectional gas holdup distribution and a rather flat liquid velocity profile are observed. If the quality of the gas distribution is poor, i.e. erratic bubble release at individual openings is observed and/or not all openings are in operation, a gas maldistribution regime is established (usually at low $U_{\mathrm{G}}$ values). Thus, the determination of the upper boundary of the gas maldistribution regime is very important.

The transition from bubbly flow to churn-turbulent flow regime is a gradual process in which a transition flow regime is formed. This regime is characterized by large flow macro-structures with large eddies and widened bubble size distribution due to the onset of bubble coalescence starting near the gas distributor. Local liquid circulation patterns are also observed in the column. The occurrence and the persistence of the transition flow regime depend largely on the uniformity and the quality of the aeration.

The transition from the bubbly flow regime (which is characterized by small and slow rising bubbles) to the churn-turbulent flow regime is caused by the formation of large fast-rising bubbles. In principle, all factors that influence the occurrence (coalescence and bubble breakup) and formation (sparger design) of these large bubbles can also influence the transition velocity. The churn-turbulent flow regime is characterized by a wide distribution of the bubble sizes and by the existence of distinctive liquid circulation due to a pronounced radial gas holdup profile. In this hydrodynamic regime, mainly coalescence and breakup events occur. Larger bubbles are formed in the vicinity of the gas 
distributor due to bubble coalescence. The churn-turbulent flow regime is further characterized by vigorous mixing and coherent structures known as bubble swarms, circulation cells, etc. It is argued that in this flow regime the gas distributor has only a minor influence. The churn-turbulent flow regime is most commonly encountered in industrial bubble columns.

The characteristic mixing length is a useful quantity describing the hydrodynamics of the liquid phase. The knowledge of the correct mixing length scale depending on the operating conditions is an important issue as it is directly linked to the mass and heat transfer performance. Kawase and Tokunaga (1991) have found that the mixing length $L$ is a function of both the column diameter $D_{c}$ and the superficial gas velocity $U_{\mathrm{G}}$.

$$
L=0.045 D_{\mathrm{c}} U_{\mathrm{G}}^{-0.38}
$$

The parameter $L$ characterizes the degree and scale of mixing. It is also associated with the distance over which a turbulent eddy retains its identity.

Nedeltchev et al. (2014) reported that in a narrow (0.15 $\mathrm{m}$ in i.d.) bubble column, the mixing length concept is applicable only in the transition flow regime. They used two independent parameters, namely the 'Kolmogorov entropy' and the 'maximum number of visits in a region', in order to identify both the lower and upper boundaries of the transition flow regime and to correlate these parameters to the mixing length.

The objective of this work is to prove the applicability of the mixing length concept (Kawase and Tokunaga, 1991) in the transition flow regime, not only in a narrow $(0.15 \mathrm{~m}$ in i.d.) bubble column, but also in a large $(0.4 \mathrm{~m}$ in i.d.) column by using a new independent statistical parameter, which can also be correlated to the mixing length. This new statistical parameter will be used for flow regime identification, as well.

\section{Definition of the New Statistical Parameter}

A new dimensionless parameter $\Phi$ is introduced in order to study the range of applicability (in terms of $U_{\mathrm{G}}$ values) of the mixing length concept. It is defined as Eq. (2).

$$
\Phi=\frac{\text { Average }}{3 A A D}
$$

In the denominator of Eq. (2), the average absolute deviation $A A D$ of the time series signal $x_{i}$ is multiplied by 3 since the cut-off length (maximum interpoint or scaling distance) in the Kolmogorov entropy $(K E)$ algorithm is set equal to 3AAD (Nedeltchev et al., 2014). In this way, the comparison between $\Phi$ and $K E$ is easier. The $K E$ values were used previously to prove the applicability of the mixing length concept in the transition flow regime (Nedeltchev et al., 2014).

The average (mean) value can be calculated as follows.

$$
\bar{x}=\frac{\sum_{i=1}^{N} x_{i}}{N}
$$

Here, $x_{\mathrm{i}}$ is each value in the time series signal, which is the gas holdup in this case.

The average absolute deviation $A A D$ of the time series signal is calculated in the standard way.

$$
A A D=\frac{\sum_{i=1}^{N}\left|x_{i}-\bar{x}\right|}{N}
$$

$A A D$ is a robust statistical estimator of the data width around the mean.

\section{Experimental Setup}

Numerous gas holdup measurements were performed in two bubble columns ( 0.15 and $0.4 \mathrm{~m}$ in i.d.) by means of a conductivity wire-mesh sensor. The sensors consisted of two electrode planes each with 24, respectively, 64 stainless steel wires. One plane of the electrodes acted as a transmitter, and the other as a receiver plane. The wire-mesh sensor technology is described in more detail in Nedeltchev et al. (2014).

Wire-mesh sensors are a proper tool for imaging of multiphase flows. The purpose of these sensors is to provide reliable gas fraction data in the cross-section of the column by using this matrix-like structure of 'multiple' local sensing points. At every sensing point, the fluid composition is determined based on an electrical measurement scheme allowing basically the discrimination between gas and liquid phase. Such sensors are currently 20 years in operation and the design of these sensors for individual applications is based on engineering tools, which consider different parameters (wire diameter, number of wires, distance between sensor planes, etc.). Several studies with quick closing valves and other measurement modalities have proven the reliability of the techniques to measure gas holdup data.

The gas holdup values were estimated in percentage [\%] and they were treated in this form by our methods of analysis. Time series of the gas holdup with 60,000 points for every $U_{\mathrm{G}}$ value were obtained by cross-sectional averaging of the matrix data. Sample gas holdup fluctuations at low gas velocity in both columns are shown in Figures 1(a) and (b). The gas holdup time series in the narrow column is more turbulent and this is reflected in the $A A D$ values: $A A D=1.180 \%$ at $U_{\mathrm{G}}=0.012 \mathrm{~m} / \mathrm{s}$ in the narrow column ( $0.15 \mathrm{~m}$ in i.d.); whereas, $A A D=0.587 \%$ at $U_{\mathrm{G}}=0.011 \mathrm{~m} / \mathrm{s}$ in the large column $(0.4 \mathrm{~m}$ in i.d.). The mean values at the same $U_{\mathrm{G}}$ are comparable: $\bar{x}=3.886 \%$ at $D_{\mathrm{c}}=0.15 \mathrm{~m}$ and $\bar{x}=3.484 \%$ at $D_{c}=0.4 \mathrm{~m}$.

The superficial gas velocity $U_{\mathrm{G}}$ was varied from 0.01 to $0.16 \mathrm{~m} / \mathrm{s}$. At each $U_{\mathrm{G}}$ value, the distribution of gas holdup in the cross-section was measured with a sampling frequency of $2,000 \mathrm{~Hz}$ over a measurement period of $30 \mathrm{~s}$.

The narrow bubble column $(0.15 \mathrm{~m}$ in i.d., clear liquid height: $2.0 \mathrm{~m}$ ) was equipped with a perforated plate distributor (14 holes with diameter $\phi 4 \times 10^{-3} \mathrm{~m}$ and an open area of $1 \%)$. The larger bubble column $(0.4 \mathrm{~m}$ in i.d., clear liquid height: $2.0 \mathrm{~m}$ ) was also equipped with a perforated plate distributor (101 holes with diameter $\phi 4 \times 10^{-3} \mathrm{~m}$ and open area 

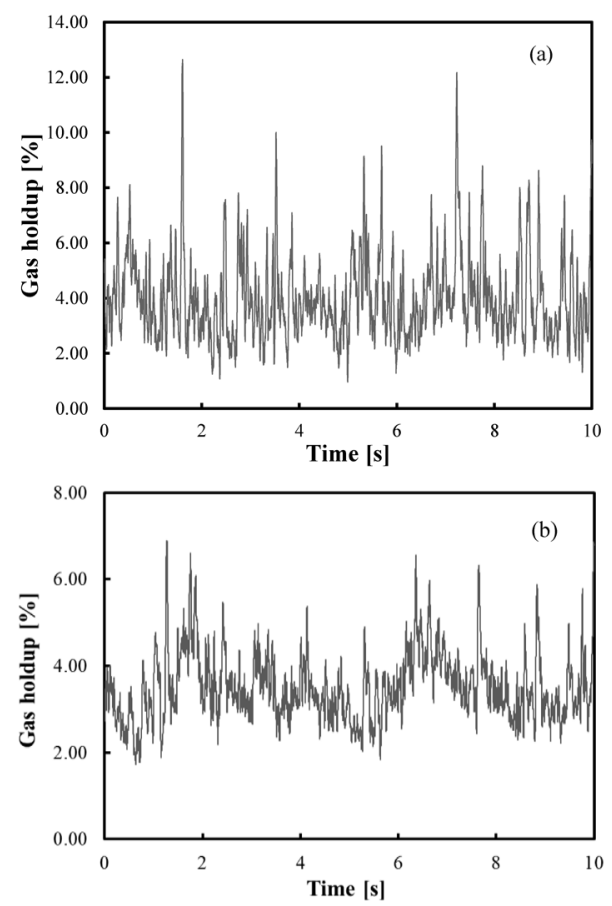

Fig. 1 Cross-sectional average gas holdup time series data $(20,000$ points equal to $10 \mathrm{~s}$ ) obtained by the wire-mesh sensor (a) $U_{\mathrm{G}}=0.012 \mathrm{~m} / \mathrm{s}$ in a narrow bubble column $(0.15 \mathrm{~m}$ in i.d.) (b) $U_{\mathrm{G}}=0.011 \mathrm{~m} / \mathrm{s}$ in a large bubble column $(0.4 \mathrm{~m}$ in i.d. $)$ operated with an air-water system

of $1 \%)$. The wire-mesh sensor was installed in both columns at a height of $1.3 \mathrm{~m}$ above the distributor plate. Air was used as the gas phase and deionized water as the liquid phase.

\section{Results and Discussion}

The new dimensionless parameter $\Phi$ (extracted from the gas holdup fluctuations) can be used not only for flow regime identification, but also for the determination of the validity of the mixing length concept. Figure 2 shows that, in a narrow $\left(0.15 \mathrm{~m}\right.$ in i.d.) bubble column at $U_{\mathrm{G}}=0.034 \mathrm{~m} / \mathrm{s}$, there is a well pronounced local peak in the $\Phi$ profile, which identifies the first transition velocity $U_{\text {trans }}$. At this $U_{\mathrm{G}}$ value, the gas maldistribution zone ends and the transition flow regime begins.

The second $U_{\text {trans }}$ value occurs at $U_{\mathrm{G}}=0.112 \mathrm{~m} / \mathrm{s}$ (see the local minimum in Figure 2) and it marks the onset of the churn-turbulent regime. In such a way, both the lower and upper boundaries of the transition flow regime in a narrow ( $0.15 \mathrm{~m}$ in i.d.) bubble column are determined on the basis of the sudden changes in the profile of the dimensionless index $\Phi$. It is noteworthy that, in the transition flow regime, the new statistical parameter $\Phi$ can be correlated successfully to the mixing length: $\Phi=62.711 \times L$ (the values of $L$ are calculated from Eq. (1)). This means that the mixing length concept is applicable in the range $0.034 \leq U_{\mathrm{G}} \leq 0.112 \mathrm{~m} / \mathrm{s}$. Such a clarification has not been provided by Kawase and Tokunaga (1991). The first $U_{\text {trans }}$ value is close to the value

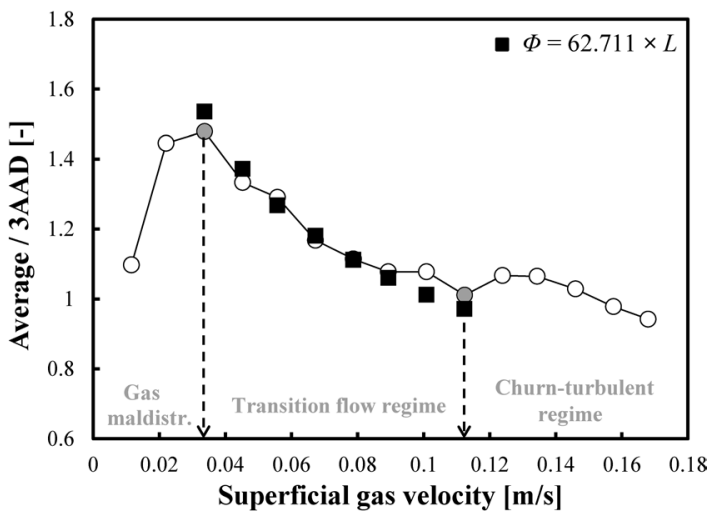

Fig. 2 Profile of the new dimensionless parameter $\Phi$ as a function of $U_{\mathrm{G}}$ in a narrow $(0.15 \mathrm{~m}$ in i.d.) bubble column operated with an air-water system

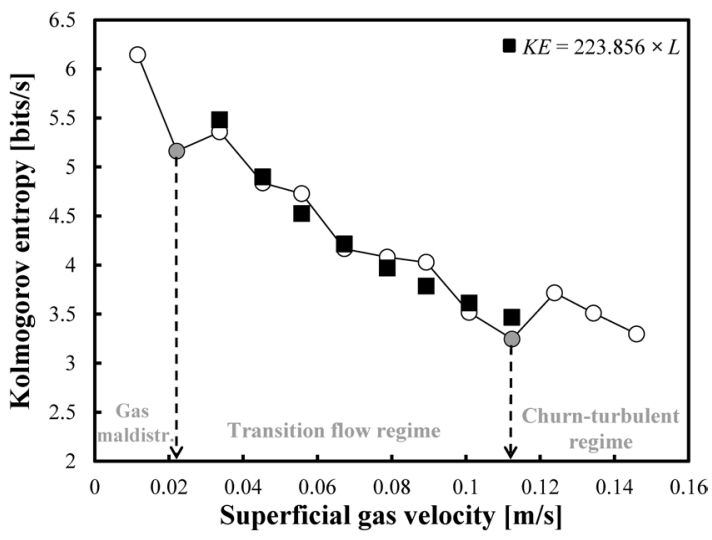

Fig. 3 Profile of the Kolmogorov entropy as a function of $U_{\mathrm{G}}$ in a narrow $(0.15 \mathrm{~m}$ in i.d.) bubble column operated with an air-water system

$(0.029 \mathrm{~m} / \mathrm{s})$ calculated for an air-water system by means of the formulas of Reilly et al. (1994).

Nedeltchev et al. (2014) identified the range of applicability of the mixing length concept on the basis of the Kolmogorov entropy $(K E)$ profile. In the case of a narrow $(0.15 \mathrm{~m}$ in i.d.) bubble column, the authors correlated the $K E$ values to the mixing length $(K E=223.856 \times L)$ in the range $0.022 \leq U_{\mathrm{G}} \leq 0.112 \mathrm{~m} / \mathrm{s}$ (see Figure 3 ). The two boundary values (identified by the local minima) indicate the end of the gas maldistribution regime and the onset of the churnturbulent regime. Nedeltchev et al. (2014) introduced also a new parameter called the 'maximum number of visits in a region' and it was also correlated to the mixing length concept in the same range (as the $K E$ values).

The profile of the new statistical index $\Phi$ in a large $(0.4 \mathrm{~m}$ in i.d.) bubble column is also capable of identifying the range of the applicability of the mixing length concept. Figure 4 shows a local peak, which identifies a change in the profile of $\Phi$ corresponding to the lower boundary of the transition flow regime at $U_{\mathrm{G}}=0.045 \mathrm{~m} / \mathrm{s}$. At $U_{\mathrm{G}}=0.101 \mathrm{~m} / \mathrm{s}$, a local minimum is observed, which identifies the onset of the churn-turbulent regime. Most likely, the local peak 


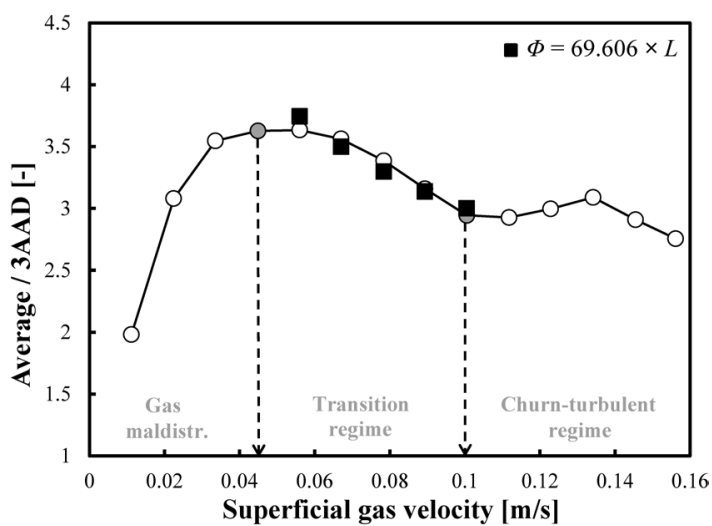

Fig. 4 Profile of the new dimensionless parameter as a function of $U_{\mathrm{G}}$ in the large bubble column $(0.4 \mathrm{~m}$ in i.d.) operated with airwater system

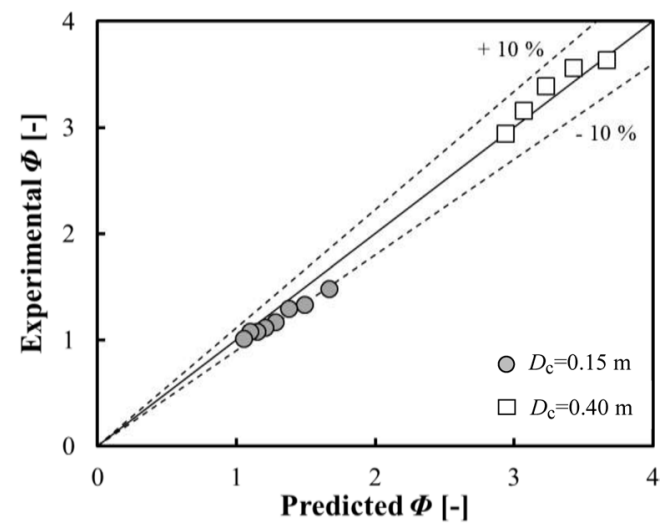

Fig. 5 Parity plot between experimental and predicted $\Phi$ values in two bubble columns operated in the transition flow regime

at $U_{\mathrm{G}}=0.134 \mathrm{~m} / \mathrm{s}$ marks the onset of the fully developed churn-turbulent flow regime. In the $U_{\mathrm{G}}$ range between the two boundaries, the new statistical index $\Phi$ can be also correlated successfully to the mixing length: $\Phi=69.606 \times L$.

It was found that the profiles of both the Kolmogorov entropy and the parameter 'maximum number of visits in a region' in the large column cannot be used for flow regime identification and for determination of the range of applicability of the mixing length concept. Only the new statistical parameter $\Phi$ can be used successfully in both bubble columns.

A comparison between the $U_{\text {trans }}$ values in both columns reveals that the first transition shifts to a somewhat higher $U_{\mathrm{G}}$ value when the column diameter enlarges; whereas, the second transition shifts to a lower $U_{\mathrm{G}}$ value. Ityokumbul (1993) found also that the first $U_{\text {trans }}$ value increases slightly with the column diameter. There is no correlation in the literature for predicting the second $U_{\text {trans }}$ value.

It is noteworthy that the proportionality constant in the correlation between $\Phi$ and $L$ in the large column is only slightly higher than the one in a narrow bubble column. This gives us confidence to propose a common constant $(\Phi=68.208 \times L)$, which is independent of the column diam- eter. The parity plot in Figure 5 shows excellent agreement between the values of the new statistical parameter $\Phi$ once determined experimentally and once from the correlation.

\section{Conclusion}

A new statistical index $\Phi$ was introduced and used for the determination of the range of applicability of the mixing length concept and for flow regime identification in two bubble columns ( 0.15 and $0.4 \mathrm{~m}$ in i.d.) operated with an airdeionized water system at ambient conditions. It was found that the first transition velocity $U_{\text {trans }}$ increases with column diameter; whereas, the second $U_{\text {trans }}$ value decreases slightly. It was possible to correlate the new parameter $\Phi$ to the mixing length $L$ only in the transition flow regime. This limitation of the range of applicability of the mixing length concept has not been described in the literature so far. A chaotic parameter (Kolmogorov entropy) and a statistical parameter ('maximum number of visits in a region') were found to be applicable for this purpose only in a narrow bubble column (0.15 $\mathrm{m}$ in i.d.).

\section{Acknowledgments}

Dr. Stoyan Nedeltchev is grateful to the European Commission for providing him with a Marie Curie Outgoing International Fellowship (7th Framework Programme, Grant Agreement No. 221832, 2010-2013). Dr. Markus Schubert gratefully acknowledges the financial support of the European Research Council (ERC Starting Grant, Grant Agreement No. 307360). The financial support of the Helmholtz Association of German Research Centres within the frame of the Initiative and Networking Fund (No. ERC-0010) is also acknowledged.

\section{Nomenclature}

$\begin{array}{llr}A A D & =\text { average absolute deviation } & {[\%]} \\ D_{\mathrm{c}} & =\text { column diameter } & {[\mathrm{m}]} \\ K E & =\text { Kolmogorov entropy } & {[\mathrm{bits} / \mathrm{s}]} \\ L & =\text { mixing length } & {[\mathrm{m}]} \\ N & =\text { number of data in a time series } & {[-]} \\ U_{\mathrm{G}} & =\text { superficial gas velocity } & {[\mathrm{m} / \mathrm{s}]} \\ U_{\text {trans }} & =\text { transition gas velocity } & {[\mathrm{m} / \mathrm{s}]} \\ x & =\text { each value in the gas holdup time series } & {[\%]} \\ \bar{x} & =\text { mean of } x \text { values } & {[\%]} \\ & & \\ \Phi & =\text { new statistical parameter (average/3AAD) } & {[-]} \\ & & \\ \text { Subscript } & \end{array}$

\section{Literature Cited}

Ityokumbul, M. T.; "Maximum Gas Velocity in Column Flotation," Miner. Eng., 6, 1279-1286 (1993)

Kawase, Y. and M. Tokunaga; "Characteristic Mixing Length in Bubble Columns," Can. J. Chem. Eng., 69, 1228-1231 (1991)

Nedeltchev, S. and A. Shaikh; "A New Method for Identification of the Main Transition Velocities in Multiphase Reactors Based on Information Entropy Theory," Chem. Eng. Sci., 100, 2-14 (2013)

Nedeltchev, S., Th. Donath, S. Rabha, U. Hampel and M. Schubert; "New Evidence for the Mixing Length Concept in a Narrow 
Bubble Column Operated in the Transition Regime," J. Chem. Eng. Japan, 47, 722-729 (2014)

Reilly, I. G., D. S. Scott, T. De Bruijn and D. MacIntyre; "The Role of Gas Phase Momentum in Determining Gas Holdup and Hydrodynamic Flow Regimes in Bubble Column Operations," Can. J.
Chem. Eng., 72, 3-12 (1994)

Shah, Y. T., B. G. Kelkar, S. P. Godbole and W.-D. Deckwer; "Design Parameters Estimations for Bubble Column Reactors," AIChE J., 28, 353-379 (1982) 